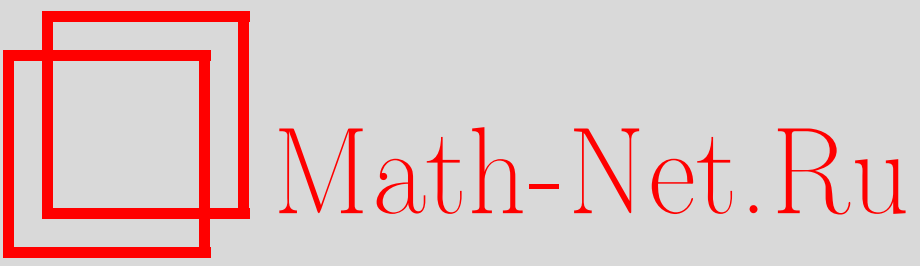

А. П. Лауринчикас, Совместная универсальность общих рядов Дирихле, Изв. РАН. Сер. матем., 2005, том 69, выпуск 1, 133-144

DOI: https://doi.org/10.4213/im627

Использование Общероссийского математического портала Math-Net.Ru подразумевает, что вы прочитали и согласны с пользовательским соглашением

http://www.mathnet.ru/rus/agreement

Параметры загрузки:

IP: 18.209 .158 .208

26 апреля 2023 г., 14:29:34 
УДК 511

\title{
А. Лауринчикас
}

\section{Совместная универсальность общих рядов Дирихле}

\author{
При некоторых условиях на коэффициенты и систему показателей получена \\ совместная теорема универсальности типа теоремы Воронина для общих рядов \\ Дирихле. \\ Библиография: 21 наименование.
}

\section{§1. Введение}

Пусть $s=\sigma+i t-$ комплексная переменная, и пусть $\mathbb{N}, \mathbb{R}$ и $\mathbb{C}$ - множества соответственно всех целых положительных, действительных и комплексных чисел. Ряд

$$
f(s)=\sum_{m=1}^{\infty} a_{m} e^{-\lambda_{m} s}, \quad \sigma>\sigma_{\mathrm{a}},
$$

называется общим рядом Дирихле. Здесь $a_{m} \in \mathbb{C},\left\{\lambda_{m}\right\}$ - возрастающая последовательность положительных чисел, $\lim _{m \rightarrow \infty} \lambda_{m}=+\infty$, а $\sigma_{\mathrm{a}}$ - абсцисса абсолютной сходимости ряда. В работе [1] была получена универсальность функции $f(s)$. Отметим, что проблема универсальности дзета-функций восходит к работам С. М. Воронина. В 1975 г. он доказал [2] (см. также [3]-[8]) замечательную теорему о приближении аналитических функций сдвигами дзета-функции Римана. Пусть при $T>0$

$$
\nu_{T}(\ldots)=\frac{1}{T} \operatorname{meas}\{\tau \in[0, T]: \ldots\},
$$

где meas $\{A\}$ означает меру Лебега множества $A \subset \mathbb{R}$, а вместо многоточия записывается условие, которому удовлетворяет $\tau$. Тогда последний вариант теоремы Воронина утверждает, что если $K$-компактноеподмножество полосы $\left\{s \in \mathbb{C}: \frac{1}{2}<\right.$ $\sigma<1\}$, обладающее связным дополнением, а функция $g(s)$ непрерывна, не имеет нулей в $K$ и аналитична внутри $K$, то для всякого $\varepsilon>0$

$$
\liminf _{T \rightarrow \infty} \nu_{T}\left(\sup _{s \in K}|\zeta(s+i \tau)-g(s)|<\varepsilon\right)>0 .
$$

Теорема универсальности, полученная для функции $f(s)$ в [1], содержит некоторые дополнительные условия. Предположим, что система показателей $\left\{\lambda_{m}\right\}$ линейно независима над полем рациональных чисел, функция $f(s)$ мероморфно продолжается на полуплоскость $\sigma>\sigma_{1}$ с некоторым $\sigma_{1}<\sigma_{\mathrm{a}}$, где все полюсы

Работа поддержана Государственным фондом науки и высшего образования Литвы. 
содержатся в компактном множестве, и $f(s)$ аналитична в полосе $D=\{s \in \mathbb{C}$ : $\left.\sigma_{1}<\sigma<\sigma_{\mathrm{a}}\right\}$. Необходимо также, чтобы при $\sigma>\sigma_{1}$ выполнялись оценки

$$
\begin{aligned}
& f(\sigma+i t) \ll|t|^{\alpha}, \quad|t| \geqslant t_{0}, \quad \alpha>0, \\
& \int_{-T}^{T}|f(\sigma+i t)|^{2} d t \ll T, \quad T \rightarrow \infty .
\end{aligned}
$$

При $x>0$ положим

$$
r(x)=\sum_{\lambda_{m} \leqslant x} 1,
$$

и пусть $c_{m}=a_{m} e^{-\lambda_{m} \sigma_{\mathrm{a}}}$. Предположим, что при $x \rightarrow \infty$

$$
\sum_{\lambda_{m} \leqslant x}\left|c_{m}\right|^{2}=\theta r(x)(1+o(1))
$$

с некоторой константой $\theta>0,\left|c_{m}\right| \leqslant d, d>0$, а также

$$
r(x)=C_{1} x^{\kappa}+B
$$

где $\kappa>1, C_{1}>0$ и $|B| \leqslant C_{2}$. Наконец, предположим, что в полуплоскости $\sigma>\sigma_{\text {a }}$ функция $f(s)$ не может быть представлена эйлеровским произведением по простым числам. Тогда имеет место следуюшее утверждение [1].

ТЕОРемА А. Пусть выполнены все сформулированные выше условия, и пусть $K$ - компактное подмножество полосы $D$, обладающее связным дополнением, а $g(s)$ - непрерывная в $K$ и аналитическая внутри $K$ функиия. Тогда для всякого $\varepsilon>0$

$$
\liminf _{T \rightarrow \infty} \nu_{T}\left(\sup _{s \in K}|f(s+i \tau)-g(s)|<\varepsilon\right)>0 .
$$

Первые результаты о совместной универсальности рядов Дирихле были получены С. М. Ворониным [7], [5], [8], а затем С. М. Гонеком [9] и Б. Багчи [10], [11]. Они доказали совместную универсальность $L$-функций Дирихле. Совместная универсальность дзета-функций Матсумото и Лерха была получена соответственно в [12] и [13]. В [14] рассматривалась совместная универсальность дзета-функций некоторых параболических форм, в [15] и [10] совместная теорема универсальности была доказана для сверток рядов Дирихле, имеюших мультипликативные коэффициенты с характером Дирихле, а в [16] - для таких сверток автоморфных $L$-функций.

Первая попытка получить совместную теорему универсальности для обших рядов Дирихле была сделана в [17]. При этом требовалось, чтобы множество $\{\log 2\} \cup \bigcup_{m=1}^{\infty}\left\{\lambda_{m}\right\}$ было линейно независимо над полем рациональных чисел, что не является естественным условием. Оказывается, что можно ограничиться только линейной независимостью системы показателей $\left\{\lambda_{m}\right\}$.

Пусть при $\sigma>\sigma_{\mathrm{a} j}$ ряд

$$
f_{j}(s)=\sum_{m=1}^{\infty} a_{m j} e^{-\lambda_{m} s}
$$

абсолютно сходится, $j=1, \ldots, n$. Как и выше, предполагаем, что функция $f_{j}(s)$ мероморфно продолжается в полуплоскость $\sigma>\sigma_{1 j}$ с некоторым $\sigma_{1 j}<\sigma_{\mathrm{aj}}$, 
все полюсы содержатся в компактном множестве, она аналитична в полосе $D_{j}=$ $\left\{s \in \mathbb{C}: \sigma_{1 j}<\sigma<\sigma_{\text {a } j}\right\}$, и что $f_{j}(s)$ в области $\sigma>\sigma_{\text {а } j}$ не может быть представлена эйлеровским произведением по простым числам, $j=1, \ldots, n$. Кроме того, при $\sigma>\sigma_{1 j}$ мы требуем выполнения оценок

$$
\begin{gathered}
f_{j}(\sigma+i t) \ll|t|^{\alpha_{j}}, \quad|t| \geqslant t_{0}, \quad \alpha_{j}>0, \\
\int_{-T}^{T}\left|f_{j}(\sigma+i t)\right|^{2} d t \ll T, \quad T \rightarrow \infty,
\end{gathered}
$$

где $j=1, \ldots, n$. Пусть

$$
c_{m j}=a_{m j} e^{-\lambda_{m} \sigma_{\mathrm{a} j}}, \quad j=1, \ldots, n,
$$

и предположим, что сушествуют $r \geqslant n$ множеств $\mathbb{N}_{k}, \mathbb{N}_{k_{1}} \cap \mathbb{N}_{k_{2}}=\varnothing$ при $k_{1} \neq k_{2}$, $\mathbb{N}=\bigcup_{k=1}^{r} \mathbb{N}_{k}$, таких, что $c_{m j}=b_{k j}$ при $m \in \mathbb{N}_{k}$, где $b_{k j}-$ некоторые комплексные числа, $k=1, \ldots, r, j=1, \ldots, n$. Пусть

$$
B=\left(\begin{array}{ccc}
b_{11} & \ldots & b_{1 n} \\
\ldots \ldots & \ldots \\
b_{r 1} & \ldots & b_{r n}
\end{array}\right)
$$

и предположим, что последовательность показателей $\left\{\lambda_{m}\right\}$ удовлетворяет соотношению (1) и

$$
\sum_{\lambda_{m} \leqslant x, m \in \mathbb{N}_{k}} 1=\kappa_{k} r(x)+B_{k}
$$

с положительной константой $\kappa_{k}$ и $\left|B_{k}\right| \leqslant C_{3}, k=1, \ldots, r$. Целью настоящей статьи является доказательство следующего утверждения.

ТЕОРемА. Пусть выполнены условия (1)-(4), система $\left\{\lambda_{m}\right\}$ линейно независима над полем рациональных чисел $u \operatorname{rank}(B)=n$. Пусть $K_{j}-$ компактное подмножество полосы $D_{j}$, обладающее связным дополнением, и функиия $g_{j}(s)$ непрерывна в $K_{j}$ и аналитична внутри $K_{j}, j=1, \ldots, n$. Тогда для всякого $\varepsilon>0$

$$
\liminf _{T \rightarrow \infty} \nu_{T}\left(\sup _{1 \leqslant j \leqslant n} \sup _{s \in K_{j}}\left|f_{j}(s+i \tau)-g_{j}(s)\right|<\varepsilon\right)>0 .
$$

\section{§2. Совместная предельная теорема в пространстве аналитических функций}

Пусть $G$ - область на комплексной плоскости. Через $H(G)$ обозначим пространство аналитических в $G$ функций, снабженное топологией равномерной сходимости на компактах. Пусть при $N>0$

$$
D_{j, N}=\left\{s \in \mathbb{C}: \sigma_{1 j}<\sigma<\sigma_{\mathrm{a} j},|t|<N\right\}, \quad j=1, \ldots, n,
$$

и

$$
H_{n, N}=H_{n}\left(D_{1, N}, \ldots, D_{n, N}\right)=H\left(D_{1, N}\right) \times \cdots \times H\left(D_{n, N}\right) .
$$


Через $\mathcal{B}(S)$ обозначим класс борелевских множеств пространства $S$. Для доказательства теоремы необходима предельная теорема в смысле слабой сходимости вероятностных мер для вероятностной меры

$$
P_{T}(A)=\nu_{T}\left(f_{1}\left(s_{1}+i \tau\right), \ldots, f_{n}\left(s_{n}+i \tau\right) \in A\right), \quad A \in \mathcal{B}\left(H_{n, N}\right),
$$

с явным видом предельной меры. Пусть $\gamma$ - единичная окружность на комплексной плоскости и

$$
\Omega=\prod_{m=1}^{\infty} \gamma_{m}
$$

где $\gamma_{m}=\gamma$ для всех $m \in \mathbb{N}$. Поскольку $\Omega$ является компактной топологической абелевой группой, на $(\Omega, \mathcal{B}(\Omega))$ сушествует вероятностная мера Хаара $m_{H}$. Это дает вероятностное пространство $\left(\Omega, \mathcal{B}(\Omega), m_{H}\right)$. Через $\omega(m)$ обозначим проекцию элемента $\omega \in \Omega$ на координатное пространство $\gamma_{m}$.

На вероятностном пространстве $\left(\Omega, \mathcal{B}(\Omega), m_{H}\right)$ определим $H_{n, N}$-значный случайный элемент $f\left(s_{1}, \ldots, s_{n} ; \omega\right)$ формулой

$$
f\left(s_{1}, \ldots, s_{n} ; \omega\right)=\left(f_{1}\left(s_{1}, \omega\right), \ldots, f_{n}\left(s_{n}, \omega\right)\right),
$$

где

$$
f_{j}\left(s_{j}, \omega\right)=\sum_{m=1}^{\infty} a_{m j} \omega(m) e^{-\lambda_{m} s_{j}}, \quad s_{j} \in D_{j, N}, \quad j=1, \ldots, n .
$$

Пусть $P_{f}$ - распределение случайного элемента $f\left(s_{1}, \ldots, s_{n} ; \omega\right)$, т. е.

$$
P_{f}(A)=m_{H}\left(\omega \in \Omega: f\left(s_{1}, \ldots, s_{n} ; \omega\right) \in A\right), \quad A \in \mathcal{B}\left(H_{n, N}\right) .
$$

Лемма 1. Вероятностная мера $P_{T}$ при $T \rightarrow \infty$ слабо сходится к мере $P_{f}$.

ДокАЗАТЕЛЬСтво. Пусть $G_{j}=\left\{s \in \mathbb{C}: \sigma>\sigma_{1 j}\right\}$, и пусть $M\left(G_{j}\right)$ - пространство мероморфных на $D_{j}$ функций, наделенное топологией равномерной сходимости на компактах $, j=1, \ldots, n$. Положим

$$
M_{n}=M\left(G_{1}, \ldots, G_{n}\right)=M\left(G_{1}\right) \times \cdots \times M\left(G_{n}\right)
$$

и определим

$$
Q_{T}(A)=\nu_{T}\left(f_{1}\left(s_{1}+i \tau\right), \ldots, f_{n}\left(s_{n}+i \tau\right) \in A\right), \quad A \in \mathcal{B}\left(M_{n}\right) .
$$

В работе [18] была получена предельная теорема для вероятностной меры $Q_{T}$. В этой теореме предполагалось, что функции $f_{1}(s), \ldots, f_{n}(s)$ удовлетворяют условиям (2) и (3), система $\left\{\lambda_{m}\right\}$ линейно независима над полем рациональных чисел и

$$
\lambda_{m} \geqslant c(\log m)^{\theta}
$$

с положительными $c$ и $\theta$. Отметим, что последнее условие вытекает из соотношения (1). Поэтому имеем, что вероятностная мера $Q_{T}$ при $T \rightarrow \infty$ слабо сходится к распределению $\left(H\left(G_{1}\right) \times \cdots \times H\left(G_{n}\right)\right)$-значного случайного элемента $F\left(s_{1}, \ldots, s_{n} ; \omega\right)$, определенного на $\left(\Omega, \mathcal{B}(\Omega), m_{H}\right)$ формулой

$$
\begin{gathered}
F\left(s_{1}, \ldots, s_{n} ; \omega\right)=\left(\sum_{m=1}^{\infty} a_{m 1} \omega(m) e^{-\lambda_{m} s_{1}}, \ldots, \sum_{m=1}^{\infty} a_{m n} \omega(m) e^{-\lambda_{m} s_{n}}\right), \\
s_{j} \in G_{j}, \quad j=1, \ldots, n .
\end{gathered}
$$


Пусть

$$
M_{n, N}=M\left(D_{1, N}\right) \times \cdots \times M\left(D_{n, N}\right) .
$$

Тогда функция $h: M_{n} \rightarrow M_{n, N}$, определенная покоординатным сужением, непрерывна, и лемма вытекает из слабой сходимости меры $Q_{T}$.

\section{§3. Носитель элемента $f\left(s_{1}, \ldots, s_{n} ; \omega\right)$}

Носителем случайного элемента $X$ назьвается носитель распределения $X$. Будем обозначать носитель элемента $X$ через $S_{X}$. Итак, в этом параграфе мы будем рассматривать носитель меры $P_{f}$ из леммы 1 . Носителем меры $P_{f}$ является минимальное замкнутое множество $S_{P_{f}}$ из $H_{n, N}$ такое, что $P_{f}\left(S_{P_{f}}\right)=1$. Множество $S_{P_{f}}$ состоит из всех таких точек $g \in H_{n, N}$, что для всякой окрестности $G$ точки $\underline{g}$ имеет место неравенство $P_{f}(G)>0$.

Мы будем пользоваться следующими утверждениями.

Лемма 2. Пусть $\left\{X_{m}\right\}$ - последовательность независимых $H_{n, N}$-значных случайных әлементов таких, что ряд

$$
\sum_{m=1}^{\infty} X_{m}
$$

сходится почти наверное. Тогда носителем суммы ряда (5) является замыкание множества всех таких $g \in H_{n, N}$, которые могут быть записаны в виде сходящегося ряда:

$$
\underline{g}=\sum_{m=1}^{\infty} \underline{g}_{m}, \quad \underline{g}_{m} \in S_{X_{m}}
$$

ДОКАЗАТЕЛЬСТВО см. в [14, лемма 5].

ЛЕмма 3. Пусть $\left\{\underline{g}_{m}\right\}=\left\{\left(g_{1, m}, \ldots, g_{n, m}\right)\right\}$ - последовательность в $H_{n, N}$, удовлетворяющая условиям:

1) если $\mu_{1}, \ldots, \mu_{n}-$ комплекснозначные меры на $(\mathbb{C}, \mathcal{B}(\mathbb{C}))$ с компактными носителями, содержащимися в $D_{1, N}, \ldots, D_{n, N}$ соответственно, такие, что

$$
\sum_{m=1}^{\infty}\left|\sum_{j=1}^{n} \int_{\mathbb{C}} g_{j, m}(s) d \mu_{j}(s)\right|<\infty
$$

mo

$$
\int_{\mathbb{C}} s^{r} d \mu_{j}(s)=0, \quad j=1, \ldots, n, \quad r=0,1,2, \ldots ;
$$

2) ряд $\sum_{m=1}^{\infty} \underline{g}_{m}$ сходится в $H_{n, N}$;

3) для любого компакта $K_{j} \subset D_{j, N}, j=1, \ldots, n$,

$$
\sum_{m=1}^{\infty} \sum_{j=1}^{n} \sup _{s \in K_{j}}\left|g_{j, m}(s)\right|^{2}<\infty .
$$

Тогда множество всех сходящихся рядов

$$
\sum_{m=1}^{\infty} a_{m} \underline{g}_{m}, \quad a_{m} \in \gamma
$$

плотно в $H_{n, N}$. 
ДОКАЗАТЕЛЬСТВО см. в [14].

Пусть

$$
w(z)=\int_{\mathbb{C}} e^{-s z} d \nu(s), \quad z \in \mathbb{C},
$$

где $\nu$ - комплекснозначная мера на $(\mathbb{C}, \mathcal{B}(\mathbb{C}))$ с компактным носителем, содержащимся в прямоугольнике

$$
D_{N}=\left\{s \in \mathbb{C}: \min _{1 \leqslant j \leqslant n}\left(\sigma_{1 j}-\sigma_{\mathrm{a} j}\right)<\sigma<0,|t|<N\right\}
$$

При изучении меры $\nu$ используются некоторые элементы теории функций экспоненциального типа.

ЛЕмма 4. Пусть $g(s)$ - целая функиия әкспоненииального типа $u\left\{u_{m}\right\}$ последовательность комплексных чисел. Кроме того, пусть $c_{1}, c_{2}$ и $c_{3}$ положительные действительные числа такие, что:

1) $\limsup _{x \rightarrow \infty} \frac{\log |g( \pm i x)|}{x} \leqslant c_{1}$;

2) $\left|u_{m}-u_{n}\right| \geqslant c_{2}|m-n|$;

3) $\lim _{m \rightarrow \infty} \frac{u_{m}}{m}=c_{3}$;

4) $c_{1} c_{3}<\pi$

Тогда

$$
\limsup _{m \rightarrow \infty} \frac{\log \left|g\left(u_{m}\right)\right|}{\left|u_{m}\right|}=\limsup _{r \rightarrow \infty} \frac{\log |g(r)|}{r}
$$

ДОКАЗАТЕЛЬСТВо см. в [19].

ЛЕмма 5. Пусть $\mu$ - комплекснозначная мера на $(\mathbb{C}, \mathcal{B}(\mathbb{C}))$ с компактным носителем, содержащимся в полуплоскости $\sigma>\sigma_{0}$,

$$
g(s)=\int_{\mathbb{C}} e^{s z} d \mu(z)
$$

$u g(s) \not \equiv 0$. Тогда

$$
\limsup _{r \rightarrow \infty} \frac{\log |g(r)|}{r}>\sigma_{0}
$$

ДОКАЗАТЕЛЬСТВО см. в [19].

ЛЕмма 6. Предположим, что существует $k, 1 \leqslant k \leqslant r$, такое, что

$$
\sum_{m \in \mathbb{N}_{k}}\left|w\left(\lambda_{m}\right)\right|<\infty
$$

Тогда

$$
\int_{\mathbb{C}} s^{l} d \nu(s)=0, \quad l=0,1,2, \ldots
$$


ДоКАЗАТЕЛЬСТВо. Из определения $w(z)$ находим, что при $x>0$

$$
|w( \pm x)| \leqslant e^{N x} \int_{\mathbb{C}}|d \nu(s)|
$$

Это показывает, что условие 1$)$ леммы 4 выполнено при $c_{1}=N$. Пусть $c_{3}-$ фиксированное число такое, что

$$
0<c_{3}<\frac{\pi}{N}
$$

Пусть $\xi$ - действительное число, удовлетворяющее условию

$$
0<4 \xi<1
$$

Пусть

$$
A=\left\{l \in \mathbb{N}: \exists r \in\left((l-\xi) c_{3},(l+\xi) c_{3}\right],|w(r)| \leqslant r^{-\kappa}\right\}
$$

Тогда

$$
\sum_{m \in \mathbb{N}_{k}}\left|w\left(\lambda_{m}\right)\right| \geqslant \sum_{l \notin A} \sum_{m}^{\prime}\left|w\left(\lambda_{m}\right)\right| \geqslant \sum_{l \notin A} \sum_{m}^{\prime} \frac{1}{\lambda_{m}^{\kappa}},
$$

где $\sum_{m}^{\prime}$ обозначает сумму по всем $m \in \mathbb{N}_{k}$, удовлетворяюшим неравенствам

$$
(l-\xi) c_{3}<\lambda_{m} \leqslant(l+\xi) c_{3}
$$

Отсюда и из условия леммы имеем

$$
\sum_{l \notin A} \sum_{\substack{m \in \mathbb{N}_{k} \\ a<\lambda_{m} \leqslant b}} \frac{1}{\lambda_{m}^{\kappa}}<\infty
$$

где $a=(l-\xi) c_{3}$ и $b=(l+\xi) c_{3}$. Суммируя по частям и применяя соотношения $(1)$ и (4), получаем, что при $l \rightarrow \infty$

$$
\begin{aligned}
\sum_{\substack{m \in \mathbb{N}_{k} \\
a<\lambda_{m} \leqslant b}} \frac{1}{\lambda_{m}^{\kappa}}= & \frac{1}{b^{\kappa}} \sum_{\substack{m \in \mathbb{N}_{k} \\
a<\lambda_{m} \leqslant b}} 1+\kappa \int_{a}^{b}\left(\sum_{\substack{m \in \mathbb{N}_{k} \\
a<\lambda_{m} \leqslant u}} 1\right) \frac{d u}{u^{\kappa+1}} \\
\geqslant & (r(b)-r(a)) \frac{\kappa_{k}}{b^{\kappa}}-\frac{2 C_{3}}{b^{\kappa}} \geqslant \frac{C_{1} \kappa_{k}}{b^{\kappa}} \\
& \times\left(\left((l+\xi) c_{3}\right)^{\kappa}-\left((l-\xi) c_{3}\right)^{\kappa}\right)-\frac{2 \kappa_{k}}{b^{\kappa}} C_{2}-\frac{2 C_{3}}{b^{\kappa}} \\
= & \frac{C_{1} \kappa_{k} c_{3}^{\kappa} l^{\kappa}}{b^{\kappa}}\left(\left(1+\frac{\xi}{l}\right)^{\kappa}-\left(1-\frac{\xi}{l}\right)^{\kappa}\right)-\frac{2 \kappa_{k} C_{2}}{b^{\kappa}}-\frac{2 C_{3}}{b^{\kappa}} \\
= & \frac{2 C_{1} \kappa_{k} c_{3}^{\kappa} l^{\kappa} \kappa \xi}{b^{\kappa} l}+O\left(\frac{1}{l^{2}}\right)-\frac{2 \kappa_{k} C_{2}}{b^{\kappa}}-\frac{2 C_{3}}{b^{\kappa}} .
\end{aligned}
$$

Отсюда, используя неравенства $\kappa>1$ и (7), получаем

$$
\sum_{l \notin A} \frac{1}{l}<\infty
$$


Предположим, что $A=\left\{a_{l}: l \in \mathbb{N}\right\}$, где $a_{1}<a_{2}<\ldots$ Тогда в силу (8) имеем

$$
\lim _{l \rightarrow \infty} \frac{a_{l}}{l}=1
$$

Ввиду определения множества $A$ сушествует последовательность $\left\{\xi_{l}\right\}$ такая, что

$$
\begin{gathered}
\left(a_{l}-\xi\right) c_{3}<\xi_{l} \leqslant\left(a_{l}+\xi\right) c_{3}, \\
\left|w\left(\xi_{l}\right)\right| \leqslant \xi_{l}^{-\kappa}
\end{gathered}
$$

Отсюда в силу соотношения (9) получаем

$$
\begin{gathered}
\lim _{l \rightarrow \infty} \frac{\xi_{l}}{l}=c_{3}, \\
\limsup _{l \rightarrow \infty} \frac{\log \left|w\left(\xi_{l}\right)\right|}{\xi_{l}} \leqslant 0 .
\end{gathered}
$$

Кроме того,

$$
\left|\xi_{m}-\xi_{n}\right|>c_{2}|m-n|
$$

с некоторым $c_{2}>0$, так как из неравенства $\left(a_{l}-\xi\right) c_{3}<\xi_{l} \leqslant\left(a_{l}+\xi\right) c_{3}$ при $n>m$ имеем, что

$$
\left|\xi_{n}-\xi_{m}\right| \geqslant\left(a_{n}-a_{m}\right) c_{3}-2 \xi c_{3} \geqslant c_{3}(n-m)-2 \xi c_{3} \geqslant c_{2}(n-m)
$$

в силу (6). Поэтому по лемме 4

$$
\limsup _{r \rightarrow \infty} \frac{\log |w(r)|}{r} \leqslant 0 .
$$

Однако из определения меры $\nu$ и леммы 5 следует, что если $w(s) \not \equiv 0$, то

$$
\limsup _{r \rightarrow \infty} \frac{\log |w(r)|}{r}>0
$$

Это противоречит неравенству (10). Поэтому $w(s) \equiv 0$, и утверждение леммы получаем, дифференщируя $w(s)$.

ЛЕмма 7. Носителем случайного әлемента $f\left(s_{1}, \ldots, s_{n} ; \omega\right)$ является все пространство $H_{n, N}$.

ДоКАЗАТЕЛЬСТво. Из определения $\Omega$ имеем, что $\{\omega(m)\}$ - последовательность независимых случайных величин, определенных на $\left(\Omega, \mathcal{B}(\Omega), m_{H}\right)$. Положим

$$
\begin{aligned}
\underline{f}_{m}\left(s_{1}, \ldots, s_{n} ; \omega(m)\right) & =\left(f_{m 1}\left(s_{1}, \omega\right), \ldots, f_{m n}\left(s_{n}, \omega\right)\right) \\
& =\left(a_{m 1} \omega(m) e^{-\lambda_{m} s_{1}}, \ldots, a_{m n} \omega(m) e^{-\lambda_{m} s_{n}}\right) .
\end{aligned}
$$


Тогда $\left\{f_{m}\left(s_{1}, \ldots, s_{n} ; \omega(m)\right)\right\}$ - последовательность независимых $H_{n, N}$-значных случайных элементов, определенных на вероятностном пространстве $(\Omega, \mathcal{B}(\Omega)$, $\left.m_{H}\right)$. Носителем каждой случайной величины $\omega(m)$ является единичная окружность $\gamma$. Поэтому множество $\left\{\underline{f}_{m}\left(s_{1}, \ldots, s_{n} ; a\right): a \in \gamma\right\}$ является носителем случайного элемента $\underline{f}_{m}\left(s_{1}, \ldots, s_{n} ; \omega(m)\right)$. Следовательно, в силу леммы 2 замыкание множества всех сходящихся рядов

$$
\sum_{m=1}^{\infty} \underline{f}_{m}\left(s_{1}, \ldots, s_{n} ; a_{m}\right), \quad a_{m} \in \gamma
$$

является носителем случайного элемента $f\left(s_{1}, \ldots, s_{n} ; \omega\right)$. Поэтому мы должны доказать, что последнее множество плотно в $H_{n, N}$. Это будет следовать из леммы 3.

Пусть $\mu_{1}, \ldots, \mu_{n}$ - комплекснозначные меры на $(\mathbb{C}, \mathcal{B}(\mathbb{C}))$ с носителями, содержашимися в $D_{1, N}, \ldots, D_{n, N}$ соответственно, такие, что

$$
\sum_{m=1}^{\infty}\left|\sum_{j=1}^{n} \int_{\mathbb{C}} a_{m j} e^{-\lambda_{m} s} d \mu_{j}(s)\right|<\infty
$$

Определим $h_{j}: \mathbb{C} \rightarrow \mathbb{C}$ формулой $h_{j}(s)=s-\sigma_{\mathrm{a} j}, j=1, \ldots, n$. Тогда

$$
\mu_{j} h_{j}^{-1}(A)=\mu_{j}\left(h_{j}^{-1} A\right), \quad A \in \mathcal{B}(\mathbb{C}),
$$

является комплекснозначной мерой на $(\mathbb{C}, \mathcal{B}(\mathbb{C}))$ с компактным носителем, содержашимся в $\widehat{D}_{j, N}=\left\{s \in \mathbb{C}: \sigma_{1 j}-\sigma_{\text {a } j}<\sigma<0,|t|<N\right\}, j=1, \ldots, n$. Отсюда и из соотношения (11) получаем

$$
\sum_{m=1}^{\infty}\left|\sum_{j=1}^{n} c_{m j} \int_{\mathbb{C}} e^{-\lambda_{m} s} d \mu_{j} h_{j}^{-1}(s)\right|<\infty
$$

где, как и вьшее, $c_{m j}=a_{m j} e^{-\lambda_{m} \sigma_{\mathrm{a}}}, j=1, \ldots, n$. Используя свойства чисел $c_{m j}$, отсюда получаем

$$
\sum_{m \in \mathbb{N}_{k}}\left|\sum_{j=1}^{n} b_{k j} \int_{\mathbb{C}} e^{-\lambda_{m} s} d \mu_{j} h_{j}^{-1}(s)\right|<\infty, \quad k=1, \ldots, r .
$$

Пусть

$$
\hat{\mu}_{k}(A)=\sum_{j=1}^{n} b_{k j} \mu_{j} h_{j}^{-1}(A), \quad A \in \mathcal{B}(\mathbb{C})
$$

и

$$
v_{k}(z)=\int_{\mathbb{C}} e^{-s z} d \hat{\mu}_{k}(s), \quad z \in \mathbb{C}, \quad k=1, \ldots, r .
$$

Тогда соотношение (12) может быть записано в виде

$$
\sum_{m \in \mathbb{N}_{k}}\left|v_{k}\left(\lambda_{m}\right)\right|<\infty, \quad k=1, \ldots, r
$$


По определению $\hat{\mu}_{k}, k=1, \ldots, r$, является комплекснозначной мерой на $(\mathbb{C}, \mathcal{B}(\mathbb{C}))$ с компактным носителем, содержащимся в $D_{N}$. Поэтому ввиду леммы 6 имеем

$$
\int_{\mathbb{C}} s^{l} d \hat{\mu}_{k}(s)=0, \quad l=0,1,2, \ldots, \quad k=1, \ldots, r
$$

Отсюда, используя определение $\hat{\mu}_{k}$ и свойства матрицы $B$, получаем

$$
\int_{\mathbb{C}} s^{l} d \mu_{j} h_{j}^{-1}(s)=0, \quad l=0,1,2, \ldots, \quad j=1, \ldots, n .
$$

Учитывая определение $h_{j}$, отсюда легко выводим, что

$$
\int_{\mathbb{C}} s^{l} d \mu_{j}(s)=0, \quad l=0,1,2, \ldots, \quad j=1, \ldots, n
$$

Ряд

$$
\sum_{m=1}^{\infty} a_{m j} \omega(m) e^{-\lambda_{m} s_{j}}
$$

для почти всех $\omega \in \Omega$ равномерно сходится на компактных подмножествах из $G_{j}$, $j=1, \ldots, n[20]$. Таким образом, сушествует последовательность $\left\{b_{m}: b_{m} \in \gamma\right\}$ такая, что ряд

$$
\sum_{m=1}^{\infty} \underline{f}_{m}\left(s_{1}, \ldots, s_{n} ; b_{m}\right)
$$

сходится в $H_{n, N}$. Также [20] при $\sigma>\sigma_{1 j}$ имеем

$$
\sum_{m=1}^{\infty}\left|a_{m j}\right|^{2} e^{-2 \lambda_{m} \sigma}<\infty, \quad j=1, \ldots, n .
$$

Поэтому свойства рядов Дирихле влекут за собой сходимость ряда

$$
\sum_{m=1}^{\infty} \sum_{j=1}^{n} \sup _{s \in K_{j}}\left|f_{m j}\left(s, b_{m}\right)\right|^{2}
$$

для всякого компакта $K_{j} \subset D_{j, N}, j=1, \ldots, n$. Отсюда, учитывая соотношение (13) и сходимость ряда (14), получаем, что все условия леммы 3 выполнены. Поэтому множество всех сходяшихся рядов

$$
\sum_{m=1}^{\infty} \hat{a}_{m} \underline{f}_{m}\left(s_{1}, \ldots, s_{n} ; b_{m}\right), \quad \hat{a}_{m} \in \gamma
$$

плотно в $H_{n, N}$. Значит, множество всех сходящихся рядов

$$
\sum_{m=1}^{\infty} \underline{f}_{m}\left(s_{1}, \ldots, s_{n} ; a_{m}\right), \quad a_{m} \in \gamma
$$

плотно в $H_{n, N}$, и лемма доказана. 


\section{§4. Доказательство теоремы}

Сначала предположим, что функции $g_{1}(s), \ldots, g_{n}(s)$ имеют аналитические продолжения соответственно в областях $D_{1, N}, \ldots, D_{n, N}$. Пусть множество $G$ состоит из $\left(y_{1}, \ldots, y_{n}\right) \in H_{n, N}$, удовлетворяющих неравенству

$$
\sup _{1 \leqslant j \leqslant n} \sup _{s \in K_{j}}\left|y_{j}(s)-g_{j}(s)\right|<\frac{\varepsilon}{4} .
$$

Очевидно, множество $G$ открыто. Поэтому из свойств слабой сходимости вероятностных мер, лемм 1 и 7 имеем

$$
\liminf _{T \rightarrow \infty} \nu_{T}\left(\sup _{1 \leqslant j \leqslant n} \sup _{s \in K_{j}}\left|f_{j}(s+i \tau)-g_{j}(s)\right|<\frac{\varepsilon}{4}\right) \geqslant P_{f}(G)>0 .
$$

Пусть функции $g_{1}(s), \ldots, g_{n}(s)$ и множества $K_{1}, \ldots, K_{n}$ удовлетворяют условиям теоремы. По теореме Мергеляна (см., например, [21]) сушествуют полиномы $p_{1}(s), \ldots, p_{n}(s)$ такие, что

$$
\sup _{1 \leqslant j \leqslant n} \sup _{s \in K_{j}}\left|p_{j}(s)-g_{j}(s)\right|<\frac{\varepsilon}{4} .
$$

Используя приведенные неравенства, имеем

$$
\liminf _{T \rightarrow \infty} \nu_{T}\left(\sup _{1 \leqslant j \leqslant n} \sup _{s \in K_{j}}\left|f_{j}(s+i \tau)-p_{j}(s)\right|<\frac{\varepsilon}{2}\right)>0 .
$$

В силу (15) получаем

$$
\left\{\tau: \sup _{1 \leqslant j \leqslant n} \sup _{s \in K_{j}}\left|f_{j}(s+i \tau)-p_{j}(s)\right|<\frac{\varepsilon}{2}\right\} \subseteq\left\{\tau: \sup _{1 \leqslant j \leqslant n} \sup _{s \in K_{j}}\left|f_{j}(s+i \tau)-g_{j}(s)\right|<\varepsilon\right\} .
$$

Отсюда и из соотношения (16) следует, что

$$
\liminf _{T \rightarrow \infty} \nu_{T}\left(\sup _{1 \leqslant j \leqslant n} \sup _{s \in K_{j}}\left|f_{j}(s+i \tau)-g_{j}(s)\right|<\varepsilon\right)>0 .
$$

Теорема доказана.

Приведем пример. Пусть $\left\{a_{m j}\right\}$ - периодическая последовательность с периодом $r \geqslant n, j=1, \ldots, n$, a $\lambda_{m}=(m+\alpha)^{\beta}$, где $\alpha$-положительное трансцендентное число и $\beta \in(0,1)$. Тогда ряд

$$
f_{j}(s)=\sum_{m=1}^{\infty} a_{m j} e^{-(m+\alpha)^{\beta} s}
$$

абсолютно сходится при $\sigma>\sigma_{\mathrm{a} j}=0, j=1, \ldots, n$, а $c_{m j}=a_{m j}$ является константой на множестве $\mathbb{N}_{k}=\{m \in \mathbb{N}: m \equiv k(\bmod r)\}$. Кроме того,

$$
r(x)=\sum_{(m+\alpha)^{\beta} \leqslant x} 1=x^{1 / \beta}+O(1) .
$$

Понятно, что элементы $b_{k j}$ можно выбрать так, чтобы $\operatorname{rank}(B)=n$. Поэтому, предполагая аналитическое продолжение функции $f_{j}(s)$ на полуплоскость $\sigma>\sigma_{1 j}$, $\sigma_{1 j}<\sigma_{\mathrm{a} j}, j=1, \ldots, n$, и выполнение оценок (2) и (3), получаем универсальность набора $f_{1}(s), \ldots, f_{n}(s)$.

Автор благодарит проф. С. В. Конягина за ценные замечания. 


\section{Список литературы}

1. Laurinčikas A., Schwarz W., Steuding J. The universality of general Dirichlet series // Analysis. 2003. V. 23. P. 13-26.

2. Воронин C.M. Теорема об "универсальности" дзета-функции Римана // Изв. АН СССР. Сер. матем. 1975. Т. 39. № 3. С. 475-486.

3. Воронин C.M. Теорема о распределении значений дзета-функции Римана // ДАН CCCP. 1975. T. 221. № 4. C. 771.

4. Воронин C. М. Исследование поведения дзета-функции Римана: Дис.... канд. физ.-мат. наук. М.: МИАН СССР, 1972.

5. Воронин С. М. Аналитические свойства производящих функций Дирихле арифметических объектов: Дис. ... д д-ра физ.-мат. наук. М.: МИАН СССР, 1977.

6. Воронин С. М. Аналитические свойства производящих функций Дирихле арифметических объектов // Матем. заметки. 1978. Т. 24. №6. С. 897-884.

7. Воронин C.M. О функциональной независимости $L$-функций Дирихле // Acta Arith. 1975. V. 27. № 2. P. 493-503.

8. Воронин C. М., Карацуба А. А. Дзета-функция Римана. М.: Физматлит, 1994.

9. Gonek S.M. Analytic properties of zeta and $L$-functions: $\mathrm{Ph}$. D. Thesis. University of Michigan, 1979.

10. Bagchi $B$. The statistical behaviour and universality properties of the Riemann zeta-function and other allied Dirichlet series: Ph. D. Thesis. Calcutta: Indian statistical Institute, 1981.

11. Bagchi B. Joint universality theorem for Dirichlet $L$-functions // Math. Z. 1982. V. 181. P. 319-334.

12. Laurinčikas A. On the zeros of linear combinations of the Matsumoto zeta-functions // Liet. Matem. Rink. 1998. V. 38. № 2. P. 185-204.

13. Laurinčikas A., Matsumoto K. The joint universality and the functional independence for Lerch zeta-function // Nagoya. Math. J. 2000. V. 157. P. 211-227.

14. Laurinčikas A., Matsumoto K. The joint universality of zeta-function attached to certain cusp forms // Proc. Sci. Seminar Faculty of Phys. and Math. Siauliai University. 2002. V. 5. P. 57-75.

15. Šleževičiene $R$. The joint universality for twists of Dirichlet series with multiplicative coefficients by characters // Analytic and Probab. Methods in Number Theory. Proc. of the Third Palanga Conf. (2001) / Eds. A. Dubickas et al. Vilnius: TEV, 2002. P. 309-319.

16. Laurinčikas A., Matsumoto $K$. The joint universality of twisted automorphic $L$-functions // J. Math. Soc. Japan. 2004. V. 56. № 3. P. 923-939.

17. Laurinčikas A. The joint universality for general Dirichlet series // Ann. Univ. Sc. Budapest. Sect. Comp. 2003. V. 22. P. 235-251.

18. Genys J., Laurinčikas A. On joint limit theorem for general Dirichlet series // Nonlinear Analysis: Modeling and Control. 2003. V. 8. № 2. P. 27-39.

19. Laurinčikas A. Limit Theorems for the Riemann Zeta-Function. London: Kluwer Academic Publishers, 1996.

20. Laurinčikas A., Schwarz N., Steuding J. Value distribution of general Dirichlet series. III // Analytic and Probab. Methods in Number Theory. Proc. of the Third Palanga Conf. (2001) / Eds. A. Dubickas et al. Vilnius: TEV, 2002. P. 137-156.

21. Walsch J.L. Interpolation and Approximation by Rational Functions in the Complex Domain // Amer. Math. Soc. Coll. Publ. 1960. V. 20.

Вильнюсский университет 